\title{
Flow-induced dilatation in isolated resistance arteries from control and streptozotocin-diabetic rats
}

\author{
R.M. Tribe ${ }^{1}$, C. R. Thomas ${ }^{2}$, L. Poston ${ }^{1}$ \\ ${ }^{1}$ Fetal Health Research Group, Department of Obstetrics and Gynaecology, Division of Medicine, UMDS, St. Thomas' Hospital, \\ London, UK \\ ${ }^{2}$ Department of Endocrinology, Diabetes and Metabolic Medicine, Division of Medicine, UMDS, St. Thomas' Hospital, London, \\ UK
}

\begin{abstract}
Summary Acetylcholine-induced vasodilatation is impaired in animal models of insulin-dependent diabetes mellitus (IDDM), and may result from altered nitric oxide synthesis or release. The response to intraluminal flow, a more physiologically relevant stimulus for nitric oxide release, is unknown. This study examined flow-induced responses in isolated resistance arteries from male Sprague-Dawley control and streptozotocin-diabetic $(45 \mathrm{mg} / \mathrm{kg}$ i.v, 4 week duration) rats. Mesenteric arteries (4--5th order) were dissected and cannulated on a pressure myograph (mean internal diameter \pm SEM at $40 \mathrm{mmHg}$, control $223 \pm 8, n=9$ vs diabetic $239 \pm 12 \mu \mathrm{m}, n=8$, NS). Arteries were preconstricted with noradrenaline $(1 \mu \mathrm{mol} / \mathrm{l})$ and intraluminal pressure raised and main-
\end{abstract}

tained at $80 \mathrm{mmHg}$. Luminal flow was raised in incremental steps $(0--1.27 \mu \mathrm{l} / \mathrm{s})$. Arteries from control animals dilated to flow while arteries from diabetic animals constricted (\% change in internal diameter \pm SEM at $0.79 \mu \mathrm{l} / \mathrm{s}$ : control $13.46 \pm 6.52, n=9$ vs diabetic - $7.44 \pm 3.38 \%, n=8 ; p<0.005)$. Incubation with $\mathrm{N} \omega$-nitro-L-arginine methyl ester $(0.1 \mathrm{mmol} / \mathrm{l})$ abolished flow responses in arteries from controls but not from diabetic rats. In conclusion, impaired flow-induced nitric oxide-mediated vasodilatation may contribute to vascular disease in IDDM. [Diabetologia (1998) 41: 34--39]

Keywords Shear stress, diabetes mellitus, endothelium, nitric oxide, vascular smooth muscle.
Vascular complications account for most of the morbidity and mortality associated with insulin-dependent diabetes mellitus (IDDM). The function of the vascular endothelium is impaired in IDDM and it is proposed that this may contribute to disease of the larger arteries and of the microvasculature [1--5]. Among the recognised endothelial defects is the impaired vasodilatory response to acetylcholine (ACh) which is generally attributed to altered nitric oxide

Received: 18 June 1997 and in final revised form: 12 September 1997

Corresponding author: Dr. R. M. Tribe, Fetal Health Research Group, Department of Obstetrics and Gynaecology, St. Thomas' Hospital, Lambeth Palace Road, London SE1 7EH, UK Abbreviations: IDDM, Insulin dependent diabetes mellitus; NO, nitric oxide; ACh, acetylcholine; STZ, streptozotocin; NA, noradrenaline; PSS, physiological salt solution; i. d., internal diameter; L-NAME, N $\omega$-nitro-L-arginine methyl ester.
(NO) synthesis or release from the endothelium [3]. Poor responses to ACh may not be a generalised defect of agonist-mediated NO release as relaxation to another potent endothelium-dependent vasodilator, bradykinin, is unaltered in isolated arteries from both diabetic patients and animal models of IDDM $[6,7]$. Moreover, the relevance of the impaired response to ACh in vitro to the in vivo control of vascular tone has been questioned, as ACh is not thought to play an important physiological vasodilatory role in most circulations. In order to evaluate endothelial dilator function in IDDM, it may be more relevant to investigate endothelial dilator responses to other, more physiological stimuli, such as blood flow. As blood flows through the lumen of the artery it exerts a frictional force, shear stress, on the endothelial cell layer which, in turn, stimulates the release of the vasodilators NO and prostacyclin $[8,9]$.

Few studies have investigated the vascular response to flow in resistance arteries in diabetes, 
although the non-invasive technique of high resolution Doppler ultrasound has recently been used to evaluate changes in conduit artery diameter in response to flow (generated by a downstream reactive hyperaemia) in diabetic patients $[10,11]$. Zenere et al. [11] demonstrated impaired flow-induced dilatation in femoral arteries of normoalbuminuric and microalbuminuric IDDM patients, but the endothelium-dependence of this flow-response was not quantified.

The aim of the present study was to investigate flow-responses in isolated small arteries from control and streptozotocin(STZ)-diabetic rats, using the technique of perfusion myography.

\section{Materials and methods}

Induction of experimental diabetes. Diabetes was induced in male Sprague-Dawley rats (250--300 g, Bantin and Kingman Ltd., Aldbrough, Humberside, UK) by caudal intravenous injection of STZ $(45 \mathrm{mg} / \mathrm{kg})$ dissolved in citrate buffer $(0.1 \mathrm{~mol} / \mathrm{l}$ trisodium citrate, $0.1 \mathrm{~mol} / \mathrm{l}$ citric acid). Development of diabetes was confirmed by the presence of glycosuria $72 \mathrm{~h}$ after injection. No attempt was made to control hyperglycaemia by insulin treatment. After 28 days, animals were killed by inhalation of $\mathrm{CO}_{2}$ and cervical dislocation. Cardiac puncture was performed to obtain blood samples for the measurement of plasma glucose (diabetic rat plasma glucose: $43.6 \pm 2.6 \mathrm{mmol} / 1$, $n=8$; enzymatic UV test, using a HK/G6P-DH method, Cobas Fara Centrifugal analyser, Roche Diagnostic Systems, Welwyn Garden City, Herts., UK). Control rats were housed separately from the diabetic animals and all animals were given food and water ad libitum. The body weight of diabetic animals was less than that of controls at 28 days (control 369.0 $\pm 7.0, n=9$ vs diabetic $276.5 \pm 10.5 \mathrm{~g}, n=8, p<0.01)$. This work was approved by the British Home Office (Project License: PPL 90/ 00764) and followed the "Principles of Laboratory Animal Care" " (NIH Publication No. 85--23, revised 1985).

Assessment of vascular function. The small intestine and intact mesentery were removed from the animal and placed in cold physiological salt solution (PSS). Branches of the mesenteric tree which run parallel to the gut wall (approximately $2 \mathrm{~mm}$ in length) were dissected free of connective tissue and mounted in a pressure myograph (Living Systems Instrumentation Inc., Burlington, V.T., USA). This technique has been described previously in detail [12--15]. Briefly, the vessel was cannulated on a pair of opposing glass microcannulae (cannulae tip diameter approximately $70 \mu \mathrm{m}$; previously matched for resistance to flow) and secured at each end with fine nylon sutures. The diameter of the cannula tip was similar to that of the vessel before being pressurised. Each vessel was orientated to the direction of flow in vitro in a similar manner to that experienced in vivo. The position of the distal cannulae was carefully adjusted to eliminate buckle, but avoiding undue stretch, of the artery. The vessel was perfused externally $(5 \mathrm{ml} / \mathrm{min})$ and intraluminally with PSS. Intraluminal flow was generated by a mini peristaltic pump and windkessels were attached on both sides of the vessel to dampen pulsatile flow. 'In line' pressure transducers monitored the proximal and distal pressure at each end of the vessel and a servo-controlled pump maintained the required mean intraluminal pressure. The vessel was visualized continuously by a CCD camera attached to a light mi- croscope $(\times 10)$ and displayed on a video monitor. The video camera was aligned so that the vessel axis was perpendicular to the horizontal scan lines, which were positioned at the same point of the artery for the duration of the experiment. Internal diameter (i.d) was measured continuously by a video dimension analyser (minimum measurable change in i.d is $1 \mu \mathrm{m}$ ), and both i.d. and pressure (proximal to the artery) recorded on a chart recorder. The artery i.d. was recorded at the end of each experimental interval.

Experimental protocol. After cannulation, the vessel was pressurised to $40 \mathrm{mmHg}$ and allowed to equilibrate for $40 \mathrm{~min}$ (superperfused continuously with PSS). Artery viability was assessed by constriction to extraluminal application of noradrenaline $(\mathrm{NA}, 1 \mu \mathrm{mol} / \mathrm{l})$ in potassium $(64 \mathrm{mmol} / \mathrm{l})$-substituted PSS and subsequent dilatation to ACh $(1 \mu \mathrm{mol} / \mathrm{l})$. All successfully mounted arteries proved viable. The vessel was pressurised to $80 \mathrm{mmHg}$ and i.d recorded before and after a $15 \mathrm{~min}$ equilibration period. The artery was then preconstricted with NA $(0.1--1 \mu \mathrm{mol} / 1)$ in PSS, to achieve an approximate $40 \%$ reduction in i.d. Once the NA-induced tone demonstrated a stable baseline, flow was initiated in incremental flow steps every $3 \mathrm{~min}(0.00--1.27 \mu \mathrm{l} / \mathrm{s})$ and changes in i. d. recorded at the end of each flow step. The flow pump had been previously calibrated and the relationship between proximal pressure and flow rate found to be linear over this range. Mean intraluminal pressure was maintained $80 \mathrm{mmHg}$ throughout the flow protocol by the servo-controlled pump, although occasionally it was necessary to make adjustments for small changes in proximal perfusion pressure which occurred as flow was increased. In the absence of flow, the vessel was superperfused with PSS for 10 min and then with $\mathrm{N} \omega$-nitro-L-arginine methyl ester ( $\mathrm{L}-$ NAME $)$ in PSS $(0.1 \mathrm{mmol} / \mathrm{l})$ for $20 \mathrm{~min}$. The vessel was then again preconstricted with NA and the flow protocol repeated in the continuous presence of L-NAME. Reproducibility of the repeated flow response was ascertained in a separate group of arteries (maximum response to flow in first flow protocol $9.5 \pm 1.8$ vs second flow protocol $8.6 \pm 1.7 \%, n=8 \mathrm{p}=\mathrm{NS}$ ).

Calculation of wall shear stress. Wall shear stress (WSS, dyn/ $\mathrm{cm}^{2}$ ) was calculated from the equation: $4 \eta ; \mathrm{Q} \times 10^{9} / \pi \mathrm{r}^{3}$, where $\eta$ is viscosity at $37^{\circ} \mathrm{C}$ (poise $=\mathrm{dyn} \cdot \mathrm{s}^{-1} \cdot \mathrm{cm}^{-2}$ ), Q is flow rate $(\mu \mathrm{l} / \mathrm{s})$ and $r$ is artery internal radius $(\mu \mathrm{m})$. The multiplication factor $10^{9}$ corrects for the use of both $\mu \mathrm{l} / \mathrm{s}$ for flow and $\mu \mathrm{m}$ for arterial radius $\left(1 \mu \mathrm{l}=10^{9} \mu \mathrm{m}^{3}\right)$; viscosity was 0.7 centipoise.

Chemicals and solutions. Chemicals used in this study were: NA (Sanofi Winthrop Ltd., Guildford, Surrey, UK); ACh (Sigma Aldrich Co. Ltd., Poole, Dorset, UK); STZ (a gift from Dr. N MacLeod, UpJohn Co., Kalamazoo, Mich., USA). All other chemicals were obtained from Merck Ltd., Poole, Dorset, UK). PSS was composed of: (mmol/l) $119 \mathrm{NaCl}, 4.7 \mathrm{KCl}, 2.5$ $\mathrm{CaCl}_{2}, 1.17 \mathrm{MgSO}_{4}, 25 \mathrm{NaHCO}_{3}, 1.18 \mathrm{NaH}_{2}, \mathrm{PO}_{4}, 0.026$ EDTA, and 5.5 glucose; $\mathrm{pH} 7.4,37^{\circ} \mathrm{C}$, gassed with $5 \% \mathrm{CO}_{2} /$ $95 \% \mathrm{O}_{2}$. In potassium-substituted PSS (64 mmol/l), equimolar $\mathrm{KCl}$ replaced $\mathrm{NaCl}$.

Statistical analysis. All values are given as mean \pm SEM. Changes in i.d. are expressed as a percentage of the initial i.d. recorded in absence of flow. The average change in i.d. over all flow steps was calculated for each artery to give a single summary measure [16] and a paired or unpaired Student's $t$-test used to compare the means of the summary measures. Significance was assumed at $p$ less than 0.05 . 


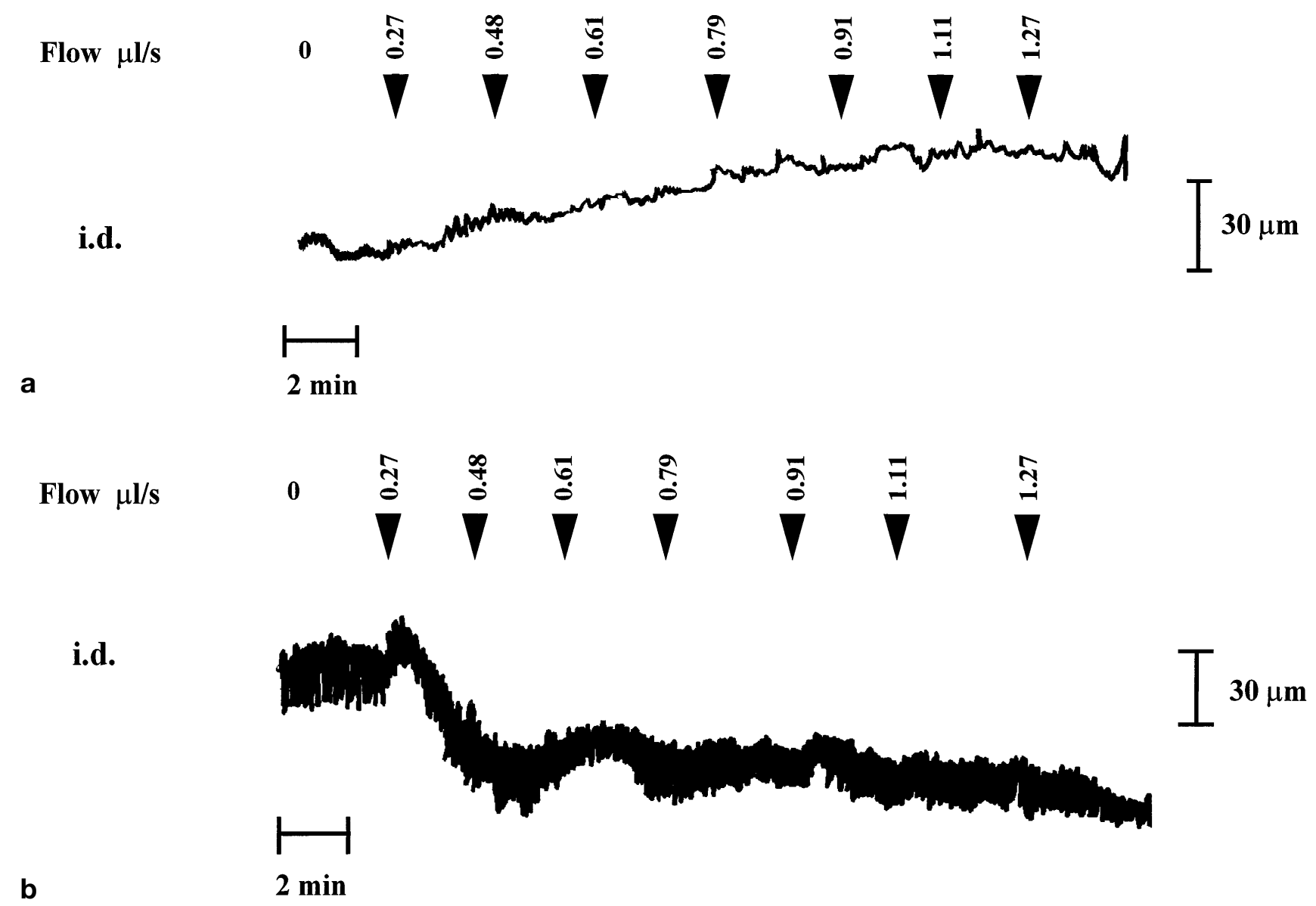

Fig. 1 a,b. Representative traces to demonstrate the changes in internal diameter in response to incremental increases in flow $(0--1.27 \mu \mathrm{l} / \mathrm{s})$ in 5 th order mesenteric arteries preconstricted with NA from: a) control and b) diabetic rat. Arteries were pressurised to $80 \mathrm{mmHg}$ and the intraluminal pressure gradient was maintained throughout the flow protocol

\section{Results}

There was no significant difference in the i.d. of arteries from control $(223 \pm 8 \mu \mathrm{m}, n=9)$ and diabetic rats $(239 \pm 12 \mu \mathrm{m}, n=8)$ when pressurised to $40 \mathrm{mmHg}$. After a $15 \mathrm{~min}$ equilibration period at $80 \mathrm{mmHg}$, the mean i.d. of arteries from the diabetic animals was slightly greater than that of controls (control $224 \pm 14$ vs diabetic $265 \pm 12 \mu \mathrm{m}, p<0.05$ ). Only arteries from control animals demonstrated development of tone during the $15 \mathrm{~min}$ period at $80 \mathrm{mmHg}$. The degree of preconstriction achieved with NA was similar between the two groups (control $54.5 \pm 5.3$ vs diabetic $52.8 \pm 3.8 \%$; NS) and the i.d. of the constricted arteries were not significantly different (control $131 \pm 7$ vs diabetic $145 \pm 11 \mu \mathrm{m}$ ).

Response to flow in arteries from controls. When pressurised to $80 \mathrm{mmHg}$, a graded increase in luminal flow was associated with dilatation in arteries from the control animals (Figs. 1 and 2). Arteries dilated maximally by $13.46 \pm 6.52 \%$ of the initial preconstricted artery diameter at a flow rate of $0.79 \mu \mathrm{l} / \mathrm{s}$ (Fig.2). Wall shear stress ranged from 0.0 to 38.6 $\mathrm{dyn} / \mathrm{cm}^{2}$ with maximum dilatation elicited at a shear stress of $20.72 \pm 3.29 \mathrm{dyn} / \mathrm{cm}^{2}$. At higher flow rates, a transient flow-induced constriction was observed.

This dilatation to flow was inhibited by preincubation of arteries with the NO synthase inhibitor LNAME, and the flow stimulus led to a significant vasoconstriction (Fig. 2, control summary measure $10.46 \pm 3.24$ vs control + L-NAME $--9.69 \pm 4.70$, $p<0.005)$. L-NAME reduced artery diameter in vessels before preconstriction with NA (\% change in i.d. --9.88 \pm 5.04 ), but this response did not achieve significance.

Response to flow in arteries from diabetic animals. In contrast to arteries from control rats, arteries from diabetic animals did not dilate to flow, indeed the same flow stimulus that evoked a dilatation in control arteries elicited a significant vasoconstriction in the arteries from diabetic animals (\% change in diameter at a flow rate of $0.79 \mu \mathrm{l} / \mathrm{s},--7.44 \pm 3.38, n=8$; summary measure for controls $10.46 \pm 3.24$ vs diabetics $--6.82 \pm 3.10, p<0.005$, Fig. 2 ). The degree of vasoconstriction to flow was similar to that observed in 


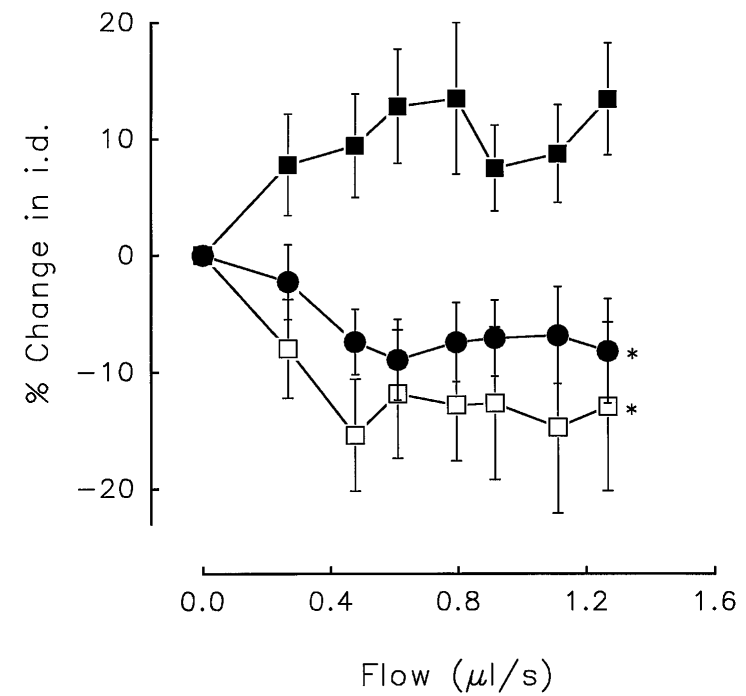

Fig. 2. Responses to flow in preconstricted small mesenteric arteries from control rats $(\square$, in PSS, and $\square$ in the presence of $0.1 \mathrm{mmol} / \mathrm{l} \mathrm{L}-\mathrm{NAME}, n=9)$, and STZ diabetic rats $(\mathbf{O}$, $n=8)$. Intraluminal flow was increased, in incremental steps, every $3 \mathrm{mins}$, from $0--1.27 \mu \mathrm{l} / \mathrm{s}$ and intraluminal pressure maintained at $80 \mathrm{mmHg}$ throughout the flow protocol. ${ }^{*} P<0.005$ compared to arteries from controls

the control arteries when in the presence of $\mathrm{L}$ NAME. There was no significant effect of L-NAME on the response of arteries from diabetic animals to flow (summary measure: diabetic - $-6.82 \pm 3.10$ vs diabetic + L-NAME $2.27 \pm 5.31)$ or on basal tone ( $\%$ change in i.d. --5.24 \pm 3.25 ).

The shear stress values generated by flow were variable and numerically greater in arteries from diabetic animals (range 0.00--65.76 $\pm 28.32 \mathrm{dyn} / \mathrm{cm}^{2}$ ) but this difference did not achieve statistical significance. The relation between shear stress and subsequent change in artery i.d. (Fig. 3) clearly reflects the insensitivity of arteries from diabetic animals to the shear stimulus.

\section{Discussion}

This study clearly demonstrates that flow elicits a significant vasodilatation in preconstricted resistance arteries from control male Sprague-Dawley rats, a response that is dependent on NO. In contrast, arteries of the STZ-diabetic rat exhibit an impaired response to flow, indeed these arteries constricted to the same flow stimulus.

Flow-induced dilatation has been studied extensively in conduit and cerebral arteries both in vivo and in vitro [9, 17--21]. There are fewer studies of this phenomenon in the resistance vasculature, although flow-induced dilatation has been observed in small mesenteric arteries in vivo [22], isolated small mesenteric arteries from pregnant rats [14], resistance sized arteries from rat skeletal muscle [23--25],

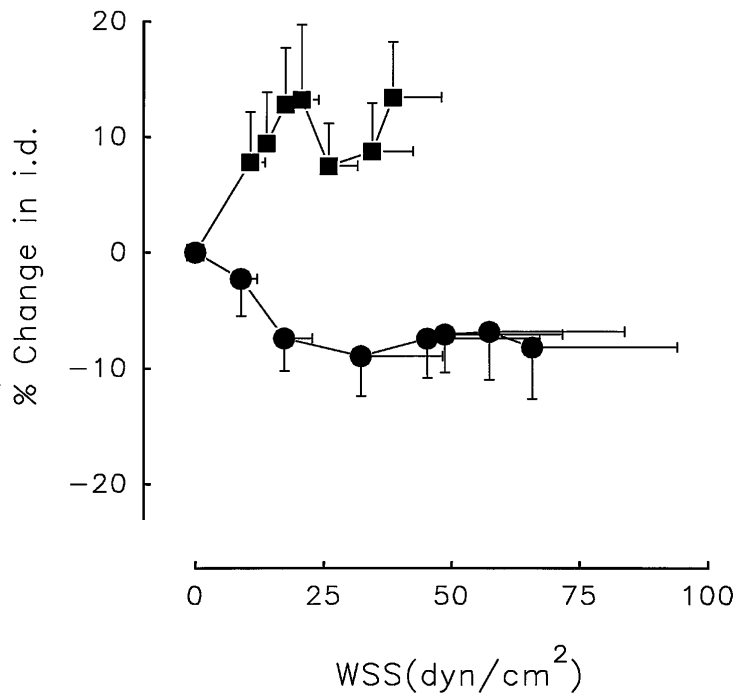

Fig. 3. Shear stress-associated changes in internal diameter. Relation between shear stress and percentage change in i.d. in response to an increasing flow stimulus in mesenteric arteries from control $(\square, n=9)$ and STZ-diabetic Sprague-Dawley rats $(\bigcirc, n=8)$. Shear stress was calculated using the equation described in the methods section

rabbit ear arteries [26] and small coronary vessels [27]. The maximum flow-induced dilatation has varied greatly between studies, an observation which may reflect variation among arteries to develop and maintain spontaneous tone. Thus, mesenteric arteries are reported to develop $14 \%$ tone at an intraluminal pressure of $80 \mathrm{mmHg}$ [14] whereas gracilis arteries (which exhibit greater vasodilatation) develop approximately $60 \%$ myogenic tone [23]. The dependence of flow responses on tone has also been reported in studies of rabbit ear arteries [26] and rat skeletal arteries [25], the greater the initial tone, the more pronounced the subsequent flow-induced dilatation. Smaller arteries generally develop greater myogenic constriction to pressure and the diversity of reports among 'resistance' sized arteries may also reflect the choice of diameter of the artery studied.

In the present study, arteries were preconstricted with NA to ensure a reproducible degree of wall tone throughout the experimental protocol. The magnitude of the dilatation elicited by flow in these preconstricted control arteries $(13.4 \%)$ was comparable to that observed in non-preconstricted rat cerebral arteries [21] and mesenteric arteries from pregnant rats [14]. The response to flow was biphasic, vessels dilated at low flow rates but at higher flow rates there was a tendency for vessel diameter to decrease. This phenomenon has been observed in other vessels [21] and it can be speculated that, at increased flow rates, vessels would resist further increases in diameter and constrict towards a 'set' point diameter, a response which would in vivo be a protective mechanism to prevent over perfusion of the end organ. The 
maximum flow-induced dilatation in arteries from controls occurred at levels of shear stress that were comparable to those calculated from the measurement of erythrocyte velocity in arteries in situ [28]. The levels of flow, however, required to achieve physiological levels of shear stress were greater than those seen in vivo due to differences in the viscosity of PSS compared to that of whole blood.

Endothelium-dependence of flow-mediated dilatation has been demonstrated in most $[14,21,23,24$, 29] but not all [18] arteries studied. In this study, dilatation was predominantly NO-mediated, as the response was abolished by the NO synthase inhibitor, L-NAME. This is in agreement with previous studies from our laboratory which showed that dilatation in mesenteric arteries from pregnant rats was also inhibited by L-NAME [14] and endothelium removal [30], but contrasts with skeletal muscle arteries [23] in which flow-induced dilatation was mediated by both $\mathrm{NO}$ and prostacylin.

It is of interest that inhibition of NO synthase evoked profound flow-induced vasoconstriction. This suggests that there may be a co-release of vasoconstrictors and vasodilators in response to flow. Endothelin, a potent vasoconstrictor has been shown, in some studies [31, 32] but not all [33] to be released by the endothelial cell layer in response to increasing flow and hence shear stress and is therefore a potential candidate mediator of the response. Vasoconstrictor prostanoids such as thromboxane have also been implicated in flow-induced vasoconstriction [34]. However, Bevan and Joyce [26] have reported flow-induced constriction in rabbit ear arteries which is not endothelium-dependent and may suggest a direct effect of flow on vascular smooth muscle tone.

The vasoconstriction observed in arteries from diabetic animals, and the lack of effect of L-NAME suggest that the abnormal response to flow is due to an impairment of the NO pathway. This does not necessarily imply that the ability to synthesize or release NO is compromised per se, but could be an indication of decreased sensitivity of the underlying smooth muscle for NO, increased NO breakdown or a specific disruption of shear stress-NO signal transduction mechanisms [3]. It is unlikely that the impaired flow response can be explained by an alteration of smooth muscle sensitivity to NO, as parallel studies performed in arteries from a group of diabetic and control animals (using wire myography) did not show any impairment of the response to the endothelium independent vasodilator, sodium nitroprusside [35]. A more likely explanation is that there is increased NO breakdown or quench due to increased superoxide generation or the presence of advanced glycosylation end products, both of which can scavenge NO on release from the endothelium [3]. While the structural integrity of the vascular endothelium is maintained in rats with diabetes of 4 weeks' duration [36], there may be specific impairment of signal transduction pathways. This has been suggested by previous investigations of vascular function in human diabetic patients [6] and the STZ-diabetic rat [7], in which vasodilatation to ACh, but not bradykinin, is impaired. Although originally suggested that flow-mediated dilatation occurs via a $G_{i}$ protein [37], a recent report has suggested that flow stimulates the phosphorylation of both $G_{i}$ and $G_{q}$ proteins [38]. Specific disruption of the $G_{i}$ protein pathway, therefore, may not be inferred from the observed attenuation of flow-induced dilatation.

The observation of impaired flow-induced dilatation in arteries from diabetic arteries contrasts with the only other study of small arteries in the STZ-diabetic rat. Fujii et al. [19], despite finding abnormal vasodilatation to $\mathrm{ACh}$, reported normal flow-induced dilatation in the basilar artery measured in vivo using a cranial window in anaesthetized rats with diabetes of 6 months' duration. However, the response to flow in these cerebral arteries was shown not to be dependent on production of NO or cyclooxygenase activity [18]. While contrasting with these observations in the rat cerebral circulation, our data agree with the observations of Zenere et al [11], who demonstrated an attenuated dilatation to flow in the femoral artery of normoalbuminuric IDDM patients, and vasoconstriction in IDDM patients with microalbuminuria.

Although myogenic tone development was not formally assessed in this study, it is interesting to note that the diabetic arteries failed to constrict to an increase in intraluminal pressure from 40 to $80 \mathrm{mmHg}$. This is in agreement with a study by Hill and Ege [39], in which a reduction in myogenic tone development was reported in isolated skeletal muscle arteries from the STZ rat. It could be speculated that reduced myogenic tone development might account for poor flow-induced dilatation in arteries from the diabetic animals, as there may be little basal tone upon which dilatory stimuli may act. However, in this study, the initial tone was similar in both groups as the arteries were preconstricted with noradrenaline and the arteries from diabetic animals failed to dilate in response to flow. Indeed, this may relate to the situation in vivo as most arteries have some sympathetic tone superimposed upon basal myogenic tone.

In summary, this study has demonstrated that arteries from an animal model of IDDM, unlike those from controls, vasoconstrict to a flow stimulus. An absence of the normal flow response could contribute to decreased blood flow in the microvasculature associated with the long-term vascular complications of diabetes. Further investigations are required to determine whether abnormal NO-mediated flow-induced dilatation is due to an increase in scavenge of $\mathrm{NO}$ or 
an impairment of the signal transduction pathways involved in shear stress-mediated NO synthesis.

Acknowledgements. The authors would like to thank the British Heart Foundation for supporting this project (grant number: PG/94186) and Mr. Paul Seed, Medical Statistician, Department of Public Health Medicine, UMDS for his advice.

\section{References}

1. Cameron NE, Cotter MA (1994) The relationship of vascular changes to metabolic factors in diabetes mellitus and their role in development of peripheral nerve complications. Diab Metab Rev 10: 189--224

2. Cohen RA (1993) Dysfunction of the vascular endothelium in diabetes mellitus. Circ 87:V-67-V-76

3. Poston L, Taylor PD(1995) Endothelium-mediated vascular function in insulin-dependent diabetes mellitus. Clin Sci 88: 245--255

4. Tooke JE (1995) Microvascular function in human diabetes. Diabetes 44: 721--726

5. Tribe RM, Poston L (1996) Oxidative stress and lipids in diabetes: a role in endothelium vasodilator dysfunction. Vasc Med 1: 195--206

6. McNally PC, Watt PAC, Rimmer T, Burden AC, Hearnshaw JR, Thurston H (1994) Impaired contraction and endothelium dependent relaxation in isolated resistance vessels from patients with insulin dependent diabetes. Clin Sci 87: 31--36

7. Taylor PD, Graves JE, Poston L (1995) Selective impairment of acetylcholine mediated endothelium dependent mediated relaxation in isolated resistance arteries of the streptozotocininduced diabetic rat. Clin Sci 88: 519--524

8. Frangos JA, Eskin SG, McIntire LV, Ives CL (1984) Flow effects on prostacyclin production by cultured human endothelial cells. Science 227: 1477--1479

9. Rubanyi GM, Romero JC, Vanhoutte PM (1986) Flow-induced release of endothelium derived relaxing factor. Am J Physiol 250:H1145--H1149

10. Goodfellow J, Ramsey MW, Luddington LA et al. (1996) Endothelium and inelastic arteries: an early marker of vascular dysfunction in non insulin-dependent diabetes. BMJ 312: 744-746

11. Zenere BM, Arcardo G, Saggiani F, Rossi L, Muggeo M, Lechi A (1995) Noninvasive detection of functional alterations of the arterial wall in IDDM patients with and without microalbuminuria. Diabetes Care 18: 975--982

12. Halpern W, Osol G, Coy GS (1984) Mechanical behaviour of pressurised in vitro prearteriolar vessels determined with a video system. Ann Biomed Eng 12: 463--479

13. Halpern W, Kelly M (1991) In vitro methodology for resistance arteries. Blood Vessels 28: 245--251

14. Cockell AP, Poston L (1996) Isolated mesenteric arteries from pregnant rats show enhanced flow-mediated relaxation but normal myogenic tone. J Physiol (London) 495: 545--551

15. Learmont JG, Cockell AP, Knock GA, Poston L (1996) Myogenic and flow-mediated responses in isolated mesenteric small arteries from pregnant and nonpregnant rats. Am J Obstet Gynaecol 174: 1631--1636

16. Matthews JNS, Altman DG, Campbell MJ, Royston R (1990) Analysis of serial measurements in medical research. BMJ 300: 230--235

17. Cooke JP, Rossitch E Jr, Andon NA, Loscalzo J, Dzau VJ (1991) Flow activates an endothelial potassium channel to release an endogenous nitrovasodilator. J Clin Invest 88: 1663-1671

18. Fujii K, Heistad DD, Faraci FM (1991) Flow-mediated dilatation of the basilar artery in vivo. Circ Res 69: 697--705
19. Fujii K, Heistad DD, Faraci FM (1992) Effect of diabetes mellitus on flow-mediated and endothelium-dependent dilatation on rat basilar artery. Stroke 23: 1494--1498

20. Meredith IT, Currie KE, Anderson TJ, Roddy M-A, Ganz P, Creager MA (1996) Postischemic vasodilation in human forearm is dependent on endothelium-derived nitric oxide. Am J Physiol H1435--H1440

21. Ngai AC, Winn HR (1995)Modulation of cerebral arteriolar diameter by intraluminal flow and pressure. Circ Res 77: 832-840

22. Qiu HY, Henrion D, Levy B (1994) Alterations in flow-dependent vasomotor tone in spontaneously hypertensive rats. Hypertension 24: 474--479

23. Koller A, Sun D, Huang A, Kaley G (1994) Corelease of nitric oxide and prostaglandins mediates flow-dependent dilation of rat gracilis muscle arterioles. Am J Physiol 267:H326--H332

24. Koller A, Sun D, Kaley G (1993)Role of shear stress and endothelial prostaglandins in flow- and viscosity-induced dilatation of arterioles in vitro. Circ Res 72: 1276--1284

25. Sun, D, Huang A, Koller A, Kaley G (1995)Flow-dependent dilation and myogenic constriction interact to establish the resistance of skeletal muscle arterioles. Microcirc 2: 289--295

26. Bevan JA, Joyce EH (1990) Flow-induced resistance artery tone: balance between constrictor and dilator mechanisms. Am J Physiol 258:H663--H668

27. Kuo L, Davies MJ, Chilian WM (1990) Endothelium-dependent, flow-induced dilation of isolated coronary arterioles. Am J Physiol 259:H1063--H1070

28. Lipowsky HH (1995) Shear stress in the circulation. In: Bevan JA, Kaley G, Rubanyi GM (eds) Flow-dependent regulation of vascular function. Oxford University Press, New York, Oxford, pp 28--45

29. Cooke JP, Stamler J, Andon N, Davies PF, McKinley G, Loscalzo J (1990) Flow stimulates endothelial cells to release a nitrovasodilator that is potentiated by reduced thiol. Am J Physiol 259:H804--H812

30. Cockell AP, Poston L (1997) 17 $\beta$-estradiol stimulates flow-induced vasodilatation in isolated small mesenteric arteries from prepubertal female rats. Am J Obstet Gynecol (in press)

31. Bodin P, Milner P, Winter R, Burnstock G (1992) Chronic hypoxia changes the ratio of endothelin to ATP release from rat aortic endothelial cells exposed to high flow. Proc Royal Soc Lon B 247: 131--135

32. Kuchan MJ, Frangos JA (1993) Shear stress regulates endothelin-1 release via protein kinase $\mathrm{C}$ and cGMP in cultured endothelial cells. Am J Physiol 264:H150--H156

33. Malek AM, Greene AL, Izumo S (1993) Regulation of endothelin gene by fluid shear stress is transcriptionally mediated and independent of protein kinase C and cAMP. Proc Natl Acad Sci USA 90: 5999--6003

34. Peeters FA, Van den Bossche R, Bult H, Herman AG (1991) Thromboxane and prostacyclin production in the perfused rabbit lung. Prostaglandins Leukot Essent Fatty Acids 43: 239246

35. Tribe RM, Palmer AM, Thomas CR, Poston L (1996) Effect of vitamin $\mathrm{E}$ and vitamin $\mathrm{C}$ on vascular endothelial function in the STZ-diabetic rat. J Vasc Res 33 [Suppl 2]:23 (Abstract)

36. Nathanaliedes C, Poston L, Warley A (1996) The structure of mesenteric arteries is not altered in rats with streptozotocin induced diabetes of 4 weeks duration. J Vasc Res 33 [Suppl 2]:13 (Abstract)

37. Ohno M, Gibbons GH, Dzau VJ, Cooke JP(1993) Shear stress elevates endothelial cGMP. Role of a potassium channel and G protein coupling. Circ 88: 193--197

38. Guva SRP, Clark CB, Frangos JA (1996) Fluid flow rapidly activates $G$ proteins in human endothelial cells: involvement of $G$ proteins in mechanochemical signal transduction. Circ Res 79: 834--839 